\title{
Factors Associated with Mortality of Patients on Hemodialysis
}

\author{
Jorge Coronado $^{1^{*}}$, Benito Balmaceda ${ }^{2}$, Amaury Ariza ${ }^{3}$, Enrique Ramos ${ }^{4}$, Cindy Mestra ${ }^{4}$ and Stephany Montenegro ${ }^{4}$
}

${ }^{1}$ Internal Medicine and Nephrology, University of Cartagena, Colombia

${ }^{2}$ Department of Nephrology, University of Cartagena, Colombia

${ }^{3}$ Department of Internal Medicine, University of Cartagena, Colombia

${ }^{4}$ School of Medicine, University of Cartagena, Colombia

"Corresponding author: Coronado Jorge, Internal Medicine and Nephrology, University of Cartagena, Colombia, Tel: +573145240109; E-mail: jcoronadod@unicartagena.edu.co; jocodada@yahoo.es

Received date: October 22, 2016; Accepted date: November 26, 2016; Published date: December 03, 2016

Copyright: (c) 2016 Jorge C, et al. This is an open-access article distributed under the terms of the Creative Commons Attribution License; which permits unrestricted use; distribution; and reproduction in any medium; provided the original author and source are credited.

\begin{abstract}
Introduction: End-stage renal disease is associated with a high annual rate of cardiovascular disease mortality. To this date, there is no local research regarding this topic.
\end{abstract}

Objective: To identify risk factors associated with mortality in prevalent chronic dialysis patients.

Methods: A case-control study was conducted in 2011-2012, in the city of Cartagena, Colombia. Deceased adult patients were included in the period. Those with advanced cancer, AIDS, liver cirrhosis, Child-Pugh C or patients with incomplete records were excluded. For each case two controls matched by age, sex and dialysis shift were selected. The clinical records were reviewed to consider social and demographic data, comorbidities, clinical and paraclinical variables. Statistical analysis was performed using Chi square, Student t-Test, Mann-Whitney and Multivariate Logistic Regression Analysis.

Findings: This study chose 85 cases from 109 deceased patients and 170 controls were chosen. No statistically significant differences were found among the patients treated under the Social Security System with regards to Comorbidities, Cause of ESRD, Pre-dialysis Blood Pressure, Hemoglobin, Kt/V, Calcium, Phosphorus, Triglycerides, Ferritin and intact parathyroid hormone (PTH) levels.

Causal associations found for time variables in hemodialysis of less than one year include vascular access via catheter, anemia, total cholesterol, underweight and hypoalbuminemia. These were more frequently found in cases studied when compared to the controls. Multivariate logistic regression analysis showed that the factors associated with mortality were related to underweight (odds ratio [OR], 2.64; 95\% Confidence interval [CI], 1.04 to 6.70 ) and to hypoalbuminemia (OR, $3.0 ; 95 \% \mathrm{Cl}, 1.21$ to 7.43$)$. Traditional risk factors did not show any causal association.

Conclusion: The case control study showed that underweight and hypoalbuminemia have a statistically significant causal relationship with the factors associated with mortality on hemodialysis patients.

Keywords: Haemodialysis; End-stage renal disease; Risk factors; Mortality; Cardiovascular death

\section{Introduction}

Chronic kidney disease is considered a high-cost disease. This disease affects between $10 \%$ and $13 \%$ of the general population in Colombia and its treatment accounts for $2 \%$ of the National Health Funds expense [1]. The prevalence of end-stage renal disease with replacement therapy in adult patients has increased. By 2005 the prevalence was 305 patients per million (p.p.m.); in 2012, it increased to 621 p.p.m. [2]. The chronic kidney disease in adult patients with chronic hemodialysis therapy poses a higher risk of cardiovascular mortality which is 5 to 100 times higher than the risk of the general population. According to the world records of the Dialysis Outcomes and Practice Patterns Study (DOPPS), their mortality rates are between 10-22\% a year [3]. In Colombia, mortality associated to hemodialysis represents between 12.7 and $15.8 \%$ [2]. Cardiovascular death is the cause of $50 \%$ of such mortality [3-5]. Multiple traditional and nontraditional risk factors have been associated with high mortality rates. Traditional risk factors include: age: 65 years and older; gender: it is more frequent in men; hypertension, diabetes mellitus, dyslipidemia, smoking and cardiovascular diseases background. Non-traditional risk factors associated with dialysis include anemia, hypoalbuminemia, hyperphosphatemia, high or low cholesterol levels and hyperparathyroidism $[3,6]$. In Colombia, scanty research has been carried out on mortality in adult patients with hemodialysis therapy. One of the few studies was done by Dialysis Outcomes in Colombia (DOC). It compared patients' mortality on peritoneal dialysis vs. hemodialysis. It found a mortality risk associated to hemodialysis of $12.7 \%$ [7]. This univariate analysis demonstrated that this mortality risk was associated with patients 65 years of age and older, a disease history, diabetes and mild to moderated malnutrition [7].

Data from the Ministry of Social Protection in 2010 for the Bolivar Province and the City of Cartagena registered prevalence of stage 5 
chronic kidney disease in 56.84 and 76.8 cases per 100,000 habitants, respectively [2]. According to the last report of The Colombian High Cost Disease Funds about the chronic kidney disease situation in 2012, the city of Cartagena has a prevalence of end-stage renal disease of 823.7 p.p.m., which is higher than the national average [2]. Coronado et al. [8] studied the prevalence of cardiovascular risk factors in adult patients with hemodialysis therapy in Cartagena between 2004 and 2007. They found that $80 \%$ of the population with hemodialysis was between 55 and 69 years of age. The males had higher frequency with $55.9 \%$. In addition, $90.5 \%$ of these patients suffered hypertension, and a high percent of them (46.9\%) had pressure values out higher than the target values. Despite the frequent and programmed clinical and paraclinical work with these patients, approximately $50 \%$ of the patients studied had anemia (47.4\%). Other findings were: hypertriglyceridemia (40.8\%), HDL low levels (31.4\%) and LDL high levels (28.3\%). Malnutrition and obesity were present as well: 20.8 and $24.4 \%$, respectively [8].

Given the inherent features of the population in Cartagena, Colombia, a case-control study of prevalent adult hemodialysis patients was designed to determine sociodemographic, clinical and paraclinical characteristics of adult patients with hemodialysis, to characterize the most common causes of chronic kidney disease, to determine the most frequent causes of death and to estimate the association between non-traditional and traditional risk factors and the risk of death under the hypothesis that the presence of such risk factors predispose to increased mortality.

\section{Materials and Methods}

A case-control study was carried out with adult patients on chronic hemodialysis treatment in two of the main dialysis units in Cartagena - Colombia from the 1st of January 2011 to the 31st of December 2012. All those hemodialysis patients who died during the study period were selected as Cases and the Controls were the active hemodialysis patients during the same period. The study included all patients over 18 years of age on chronic hemodialysis (for more than three months) three times a week. The study excluded patients with advanced malignant disease, with HIV infection category C, Child Pugh C liver cirrhosis or incomplete data in the medical record.

For each case, two controls were selected (1:2). Controls were chosen randomly from the active population undergoing hemodialysis by the time of the death of the cases. They were paired according to gender and age ranges according those of the World Health Organization (WHO), and dialyzed at the same schedule (same day of the week and same time of the day) than the deceased patients. All the cases presented during the period of study from both renal units were included. For this reason, the sample size was not calculated and it was limited by the consecution of two controls for each case.

The medical history and clinical records of all the patients were available and were used to extract the following data: sociodemographic variables, personal background, comorbidities, clinical and paraclinical variables, characteristic of Hemodialysis process. Sociodemographic variables considered for this study included: age, gender and social security system. For the cases, an exact age at the moment of death was recorded and for the controls the age at the moment of the death of the respective peers from the case group.

Personal medical history and comorbidities variables included: presence of chronic hypertension, dyslipidemia (hypercholesterolemia or hypertriglyceridemia), diabetes mellitus Type 1 or 2, cardiovascular diseases (myocardial infarction, coronary disease, heart failure or arrhythmia, coronary bypass, angioplasty with and without coronary stent) cerebrovascular diseases (background of TIA, hemorrhagic and ischemic stroke), peripheral vascular disease (carotid stenosis, aorta stenosis in limbs or non-traumatic amputation) or any other pathology, and also the cause of the chronic kidney disease according to the nephrologist's criteria.

Clinical variables included were hemodialysis time for cases and controls (expressed in months of treatment until the date of case's death), type of vascular access, pre-dialysis blood pressure, dry weight, Body Mass Index, percentage of interdialytic weight gain, and morbidity and $\mathrm{Kt} / \mathrm{V}$ mortality index. Therefore, the average of the 3 months before death, and the same period for both controls were calculated. In addition, the causes of death i.e., cardiovascular, infectious, cerebrovascular or other, were also registered.

Laboratory variables included were hemoglobin, serum phosphorus and serum calcium (expressed in the average of six months (or months recorded on medical records for cases of less than 6 months) before the date of the case's death and at the same period for their control peers. Other paraclinical variables considered were albumin, intact parathyroid hormone levels, ferritin, triglycerides and total cholesterol. The value taken was the last semiannual checks before the death and at the same period for both controls.

The possibility of selection bias in this study is null since the definition of Case is an adult deceased patient that underwent chronic hemodialysis treatment, and the Control is that still active at the moment of the case's death (considering other inclusion criteria). Thus, this eliminates the chance of mistaken classification of the controls. Information bias could take place because of erroneous variable measurement by the health workers in charge of hemodialysis adult patients in the medical records. Notwithstanding, these are specialized hemodialysis units so the information of this study is considered reliable. This reduces the possibility of non-differential information bias. Last, confusion of association bias was prevented pairing controls by dialysis schedule, age and gender with the cases and the analysis was controlled by the model of multivariate logistic regression.

\section{Statistical Analysis}

Continuous variables were reported as mean or medians according to their probability distribution. Categorical variables were reported in terms of percentage. For hypothesis testing between cases and controls for qualitative variables a chi-squared test was used. For quantitative variables, $\mathrm{t}$ student or Mann-Whitney $\mathrm{U}$ Tests were used according to normality criteria. These hypothesis tests were performed with the aim of verifying the comparability between cases and controls. As for bivariate analysis, the information was recorded in contingency tables to estimate the raw Odds Ratio (OR) with 95\% confidence intervals $(95 \% \mathrm{CI})$ and consequently multivariate analysis through logistic regression to adjust the association strength of the set of variables included in the model. This included all the variables with statistically significant differences shown by the hypothesis testing, plus variables with potential deleterious effects known or suggested on previous studies. Data processing was performed using the Stata 11 Program. A value of $\mathrm{p}<0.05$ was considered statistically significant.

\section{Results}

From 109 potential cases, 85 (78\%) were included in this study. Of the cases excluded, 2 cases they did not comply with the 3-times-a- 
Citation: Jorge C, Benito B, Amaury A, Enrique R, Cindy M, et al. (2016) Factors Associated with Mortality of Patients on Hemodialysis. J Nephrol Ther 6: 275. doi:10.4172/2161-0959.1000275

Page 3 of 7

week dialysis criteria and 22 cases (20\%) had advanced cancer (18) and missing information (4). From 269 potential controls, 170 controls were chosen randomly, paired by gender, age and dialysis schedule, two per each case, for a total of 255 patients. As in Table 1, the age range for cases and controls was between 66 and 67 respectively.

The classification of age groups in the cases was: older adults: $31.8 \%$; seniors: $30.6 \%$; middle-aged adults: $28.2 \%$; older seniors: $5.9 \%$; and young adults: $3.5 \%$.

For controls it was: older adults: $41.2 \%$; middle-aged adults: $26.5 \%$; older adults: $25.8 \%$, young adults: $4.1 \%$ and older seniors $2.3 \%$. There was no statistically significant difference in age $(\mathrm{p}=0.377)$ and sex $(\mathrm{p}=0.961)$ between cases and controls.

Social Security System of cases was contributive $61.2 \%$, subsidized $34.1 \%$ and special regime $4.7 \%$; controls were contributive $68.8 \%$, subsidized $26.5 \%$ and special regime $4.7 \%$. The most frequent comorbidities present by cases were hypertension 84.7 , diabetes mellitus $45.9 \%$, ischemic heart disease $17.7 \%$, stroke $10.6 \%$ and heart failure $9.4 \%$. For the controls, these were hypertension $88.2 \%$, diabetes mellitus $39.4 \%$, ischemic heart disease $12.4 \%$, heart failure $8.2 \%$ and stroke $5.3 \%$. On the other side, the most frequent causes of end-stage renal disease for the cases were hypertensive nephropathy $50.6 \%$, diabetic nephropathy $30.6 \%$, unknown $9.4 \%$, glomerulopathy $3.5 \%$ and obstructive uropathy $1.2 \%$. For controls they were hypertensive nephropathy $45.9 \%$, diabetic nephropathy $33.5 \%$, unknown $6.5 \%$, glomerulopathy $3.5 \%$ and obstructive uropathy $3.5 \%$. No statistically significant differences were observed when comparing general characteristics, comorbidities and causes of end-stage renal disease between cases and controls (Table 1).

\begin{tabular}{|c|c|c|c|}
\hline & $\begin{array}{l}\text { Cases } \\
n=85\end{array}$ & $\begin{array}{l}\text { Controls } \\
n=170\end{array}$ & $P$ value \\
\hline Gender M & $45(52.9)$ & $92(54.1)$ & 0.9618 \\
\hline Age Ranges [RI] & 66 [58-78.5] & $67[55-76]$ & 0.3778 \\
\hline Young adults & $3(3.5)$ & $7(4.1)$ & 0.9126 \\
\hline Middle-aged adults & $24(28.2)$ & $45(26.5)$ & 0.8898 \\
\hline Older adults & $27(31.8)$ & $70(41.2)$ & 0.1867 \\
\hline Seniors & $26(30.6)$ & $44(25.8)$ & 0.5085 \\
\hline Older Seniors & $5(5.9)$ & $2(2.3)$ & 0.2656 \\
\hline \multicolumn{4}{|l|}{ Social Security } \\
\hline Contributive & $52(61.2)$ & $117(68.8)$ & 0.2848 \\
\hline Subsidized & $29(34.1)$ & $45(26.5)$ & 0.2653 \\
\hline Special regime & $4(4.7)$ & $8(4.7)$ & 0.7536 \\
\hline \multicolumn{4}{|l|}{ Comorbidities } \\
\hline Hypertension & $72(84.7)$ & $150(88.2)$ & 0.5575 \\
\hline DM & 39 (45.9) & 67 (39.4) & 0.3909 \\
\hline Ischemic Heart Disease & $15(17.7)$ & $21(12.4)$ & 0.3403 \\
\hline Stroke & $9(10.6)$ & $9(5.3)$ & 0.1944 \\
\hline Heart Failure & $8(9.4)$ & $14(8.2)$ & 0.9320 \\
\hline APE & $5(5.9)$ & $7(4.1)$ & 0.7441 \\
\hline Dyslipidemia & $1(1.2)$ & $1(0.6)$ & 0.8114 \\
\hline Others & $3(3.5)$ & $11(6.5)$ & 0.4844 \\
\hline Non present & $1(1.2)$ & $0(0.0)$ & 0.7048 \\
\hline \multicolumn{4}{|l|}{ Causes of CKD } \\
\hline Hypertensive nephropathy & $43(50.6)$ & $78(45.9)$ & 0.5650 \\
\hline Diabetic nephropathy & $26(30.6)$ & 57 (33.5) & 0.7458 \\
\hline Glomerulopathy & $3(3.5)$ & $6(3.5)$ & 0.7178 \\
\hline
\end{tabular}


Citation: Jorge C, Benito B, Amaury A, Enrique R, Cindy M, et al. (2016) Factors Associated with Mortality of Patients on Hemodialysis. J Nephrol Ther 6: 275. doi:10.4172/2161-0959.1000275

Page 4 of 7

\begin{tabular}{|l|l|l|l|}
\hline Obstructive & $1(1.2)$ & $6(3.5)$ & 0.5128 \\
\hline Polycystic Kidneys & $1(1.2)$ & $3(1.8)$ & 0.8655 \\
\hline Others & $2(2.3)$ & $4(2.3)$ & 0.6577 \\
\hline Lupus & $1(1.2)$ & $5(2.9)$ & 0.6835 \\
\hline Unknown & $8(9.4)$ & $11(6.5)$ & 0.5634 \\
\hline
\end{tabular}

Table 1: Basal characteristics of End-Stage renal disease patients with hemodialysis treatment DM: Mellitus Diabetes. APE: Acute Pulmonary Edema. CKD: Chronic Kidney Disease.

Comparing clinical and paraclinical characteristics between cases and controls, the following statistically significant differences were observed at the time of hemodialysis treatment (cases 36 months vs. controls 60 months; $\mathrm{p}=0,0076$ ), less than 12 months with dialysis treatment (cases $18.8 \%$ vs. controls $7.7 \%$; $=0,0155$ ), underweight (cases $29.4 \%$ vs. controls $13.5 \%$; $\mathrm{p}=0,0038$ ), anemia (cases $30.6 \%$ vs. controls 12.4\%; $\mathrm{p}=0,0008$ ), catheter vascular access (cases $51.8 \%$ vs. controls 25.3\%; $\mathrm{p}=0,0001$ ), total cholesterol (cases $151.9 \mathrm{mg} / \mathrm{dL}$ vs. controls $162.9 \mathrm{mg} / \mathrm{dL} ; \mathrm{p}=0,03$ ) and hypoalbuminemia (cases $42.3 \%$ vs. controls $14.1 \% ; \mathrm{p}=0,001$ ). No statistically significant differences were found in relation with morbidity and mortality cardiovascular index $\mathrm{Kt} / \mathrm{V}<1,4$, predialysis diastolic and systolic pressure, interdialytic weight gain, phosphor, calcium, Intact PTH, triglycerides and ferritin (Table 2).

\begin{tabular}{|c|c|c|c|}
\hline & Cases $(n=85)$ & Controls $(n=170)$ & $P$ value \\
\hline Hemodialysis time (years) & 36 [16.5 - 84] & $60[42-96]$ & 0.0076 \\
\hline$<1$ & $16(18.8)$ & $13(7.7)$ & 0.0155 \\
\hline $1 y<5$ & $34(40.0)$ & $67(39.4)$ & 0.9653 \\
\hline$\geq 5$ & $35(41.2)$ & $90(52.9)$ & 0.1033 \\
\hline Predialytic SBP & $150.7 \pm 23.1$ & $146.9 \pm 22.8$ & 0.2082 \\
\hline$\geq 110 y \leq 140$ & $20(23.5)$ & $56(32.9)$ & 0.1608 \\
\hline$>140$ & $60(70.6)$ & $101(59.4)$ & 0.1074 \\
\hline$<110$ & $5(5.9)$ & $13(7.7)$ & 0.7880 \\
\hline Predialytic DBP & $77.6 \pm 12.1$ & $74.8 \pm 10.3$ & 0.0592 \\
\hline$\geq 70 y \leq 90$ & $49(57.6)$ & $97(57.1)$ & 0.9536 \\
\hline$>90$ & $14(16.5)$ & $15(8.8)$ & 0.1059 \\
\hline$<70$ & $22(25.9)$ & $58(34.1)$ & 0.2351 \\
\hline Interdialytic Gain & $4.1 \pm 1.9$ & $3.8 \pm 1.4$ & 0.2146 \\
\hline Interdialytic Gain > 5.2 & $19(22.3)$ & $22(12.9)$ & 0.0806 \\
\hline $\mathrm{Kt} / \mathrm{v}$ & $1.41 \pm 0.32$ & $1.49 \pm 0.26$ & 0.0558 \\
\hline $\mathrm{Kt} / \mathrm{v}<1.4$ & $43(50.6)$ & $102(60.0)$ & 0.1954 \\
\hline \multicolumn{4}{|l|}{ Vascular Access } \\
\hline Catheter & $44(51.8)$ & $43(25.3)$ & $<0.0001$ \\
\hline Fistula & $41(48.2)$ & $127(74.7)$ & \\
\hline BMI & $21.6 \pm 4.5$ & $23.2 \pm 4.4$ & 0.0047 \\
\hline Underweight $<18.5$ & $25(29.4)$ & $23(13.5)$ & 0.0038 \\
\hline Normal weight $18.5-24.9$ & $40(47.1)$ & $91(53.5)$ & 0.4060 \\
\hline Overweight $25-29.9$ & $16(18.8)$ & $46(27.1)$ & 0.1932 \\
\hline
\end{tabular}


Citation: Jorge C, Benito B, Amaury A, Enrique R, Cindy M, et al. (2016) Factors Associated with Mortality of Patients on Hemodialysis. J Nephrol Ther 6: 275. doi:10.4172/2161-0959.1000275

Page 5 of 7

\begin{tabular}{|c|c|c|c|}
\hline Obesity $\geq 30$ & $4(4.7)$ & $10(5.9)$ & 0.9165 \\
\hline \multicolumn{4}{|l|}{ Paraclinical } \\
\hline Hemoglobin Me [RI] & $11.1[9.5-12.0]$ & $11.8[11.0-12.5]$ & 0.0002 \\
\hline Anemia & $26(30.6)$ & $21(12.4)$ & 0.0008 \\
\hline BUN & $50.2 \pm 15.4$ & $50.3 \pm 11.3$ & 0.9511 \\
\hline Albumin Me [RI] & 3.63 [3.18-3.92] & 3.96 [3.64-4.2] & $<0.0001$ \\
\hline Hypoalbuminemia & $36(42.3)$ & $24(14.1)$ & $<0.0001$ \\
\hline Phosphor & $4.1 \pm 1.2$ & $4.2 \pm 1.2$ & 0.6656 \\
\hline Hyperphosphoremia & $10(11.8)$ & $28(16.5)$ & 0.4201 \\
\hline Calcium Me [RI] & 8.63 [8.41-9.05] & $8.81[8.5-9.19]$ & 0.1169 \\
\hline Calcium (goals) & $50(58.8)$ & $103(60.6)$ & 0.8879 \\
\hline PTH Me [RI] & 234 [89-474] & $303[117-513]$ & 0.1023 \\
\hline Calcium (goals) & $50(58.8)$ & $103(60.6)$ & 0.8879 \\
\hline Ferritin Me [RI] & 450 [263.6-996.0] & 493 [290.0-903.5] & 0.8303 \\
\hline Ferritin (goals) & $37(43.5)$ & $64(37.7)$ & 0.4492 \\
\hline Triglycerides Me [RI] & 110 [81.4 - 157] & 112 [82.0 - 162] & 0.6952 \\
\hline Hypertriglyceridemia & $11(12.9)$ & $22(12.9)$ & 0.8429 \\
\hline Total Cholesterol & $151.9 \pm 40$ & $162.9 \pm 40$ & 0.0383 \\
\hline
\end{tabular}

Table 2: Clinical Characteristics of Cases and Controls. SBP: Systolic Blood Pressure. DBP: Dyastolic Blood Pressure. BMI: Body mass index. BUN: Blood Urea Nitrogen. PTH: Parathyroid hormone.

When estimating raw associations between traditional risk factors, non-traditional risk factors and mortality, causal associations were found for dialysis of less than one year (OR, 2.80; $95 \%$ CI, 1.28 to 6-14), catheter vascular access (OR, 3.13; 95\% CI, 1.83 to 5.48 ), underweight (OR, 2.66; 95\% CI, 1.40 to 5.05), anemia (OR, 3.13; 95\% CI, 1.63 to 5.99 ) and hypoalbuminemia (OR, 4.47; 95\% CI, 2.43 to 8.22). When performing multivariate logistic regression analysis, the following factors associated to mortality were found: underweight (OR, 2.64; $95 \% \mathrm{CI}, 1.04$ to 6.70 ) and hypoalbuminemia (OR, 3.0; 95\% CI, 1.21 to 7.43). No traditional risk factor showed evidence of causal association (Table 3). Among death causes, cardiovascular diseases were the most frequent cause (35.29\%), followed by infectious diseases (25.88\%), strokes $(4.7 \%)$, decompensated diabetes mellitus type 2 (3.52\%), uremia (3.52\%), encephalic cranial trauma (2.35\%). Other identified causes of death were: malnutrition, status epilepticus, noninfection acute exacerbation of chronic obstructive pulmonary disease, hepatic failure, upper gastrointestinal tract bleeding, hypoglycemia, obstructive jaundice, mesenteric ischemia, non-specific neuropathy, cholelithiasis, pulmonary thromboembolism and cervical trauma (1.17\% each one). The causes of death of $10.58 \%$ of cases remain undetermined (Table 4).

\begin{tabular}{|c|c|c|c|c|}
\hline & Raw OR & $\mathrm{Cl} 95 \%$ & Adjusted OR* & $\mathrm{Cl} 95 \%$ \\
\hline \multicolumn{5}{|l|}{ Traditional } \\
\hline Male & 0.95 & $0.57-1.61$ & 1.02 & $0.45-2.32$ \\
\hline Age $>65$ & 0.88 & $0.53-1.50$ & 0.86 & $0.40-1.83$ \\
\hline Hypertension & 0.74 & $0.35-1.57$ & 0.73 & $0.24-2.19$ \\
\hline DM & 1.30 & $0.77-2.20$ & 1.69 & $0.81-3.54$ \\
\hline Heart failure & 1.16 & $0.47-2.88$ & 1.04 & $0.28-2.66$ \\
\hline Ischemic heart disease & 1.52 & $0.73-3.13$ & 1.87 & $0.71-4.91$ \\
\hline
\end{tabular}




\begin{tabular}{|l|l|l|l|l|}
\hline Non-traditional & & & & \\
\hline Dialysis time $<1$ year & $\mathbf{2 . 8 0}$ & $\mathbf{1 . 2 8 - 6 . 1 4}$ & 0.87 & $0.28-2.66$ \\
\hline Interdialytic Gain $>\mathbf{5 . 2}$ & 1.94 & $0.98-3.82$ & 1.36 & $0.53-3.50$ \\
\hline $\mathbf{K t} / \mathbf{v}<\mathbf{1 . 4}$ & 1.46 & $0.87-2.47$ & 0.76 & $0.34-1.72$ \\
\hline Catheter vascular access & $\mathbf{3 . 1 7}$ & $\mathbf{1 . 8 3 - 5 . 4 8}$ & 1.12 & $0.49-2.55$ \\
\hline BMI < 18.5 & $\mathbf{2 . 6 6}$ & $\mathbf{1 . 4 0 - 5 . 0 5}$ & $\mathbf{2 . 6 4}$ & $\mathbf{1 . 0 4 - 6 . 7 0}$ \\
\hline Anemia & $\mathbf{3 . 1 3}$ & $\mathbf{1 . 6 3 - 5 . 9 9}$ & 1.98 & $0.71-5.53$ \\
\hline Hypoalbuminemia & $\mathbf{4 . 4 7}$ & $\mathbf{2 . 4 3 - 8 . 2 2}$ & $\mathbf{3 . 0 0}$ & $\mathbf{1 . 2 1 - 7 . 4 3}$ \\
\hline Hyperphosphoremia & 0.68 & $0.31-1.47$ & 0.67 & $0.24-1.84$ \\
\hline Calcium (goals) & 0.93 & $0.55-1.58$ & 0.75 & $0.36-1.57$ \\
\hline Hyperparathyroidism & 0.68 & $0.40-1.16$ & 0.56 & $0.26-1.22$ \\
\hline Ferritin (goals) & 1.28 & $0.75-2.17$ & 1.18 & $0.58-2.41$ \\
\hline Hypertriglyceridemia & 1.00 & $0.46-2.17$ & 1.42 & $0.45-4.43$ \\
\hline Total cholesterol higher/lower & 0.72 & $0.34-1.53$ & 0.69 & $0.23-2.08$ \\
\hline
\end{tabular}

Table 3: Raw and adjusted association considering traditional and non-traditional risk factors DM: Mellitus Diabetes. BMI: Body mass index.

\begin{tabular}{|l|l|l|}
\hline & $\mathbf{n = 8 5}$ & $\%$ \\
\hline Cardiac & 31 & 36.5 \\
\hline Infectious & 22 & 25.9 \\
\hline Cerebrovascular & 4 & 4.7 \\
\hline Others & 20 & 23.5 \\
\hline Unknown & 8 & 9.4 \\
\hline
\end{tabular}

Table 4: Causes of death.

\section{Discussion}

This case control study is one of the first initiatives to identify factors associated with mortality in prevalent patients of hemodialysis treatment in Cartagena, Colombia.

The average age of the patients was 66 years similar to the results previously provided by Coronado et al. [8] in 2009 in the city of Cartagena as consistent with the records of the High Cost Account of the Colombian Ministry of Health and Social Protection [2]. Contributive regime was the most frequent (66.7\%) in cases and controls vs. the National distribution (42.7\%). As per associated morbidities, hypertension was the most frequent (87\%), consistent with Coronado et al. [8] (90\%), however, it is higher than the National Average (57\%), the results of the DOC study and the USRDS records $2013[7,9]$. Diabetes mellitus had a frequency similar to that recorded in the DOC Study. However, it was well above the National Average [2,7].

The analysis of the most common causes of end-stage renal disease indicated that hypertension produced about half of all cases (47.5\%), followed by diabetes (32.5\%). These frequency percentages are inverted with respect to the National Data [2]. There was no statistically

significant difference in sociodemographic variables or comorbidities that could have influenced the outcome of mortality cases.

For cases, there was no statistically significant difference regarding the number of months on hemodialysis, with a high percentage being less than 1 year. This is consistent with what is well known about the mortality associated with the initiation of hemodialysis, which is higher in the first year, reaching an annual mortality rate of up to 268.8 cases/1000 patient-year according to the latest statistics from the USRDS 2013 [9].

Hypoalbuminemia and underweight were the factors associated with mortality found in the present study. They comprise the proteinenergy malnutrition complex associated with dialysis, whose genesis lies in the persistent state of chronic inflammation typical of patients on hemodialysis [10-14]. Hypoalbuminemia is one of the nontraditional risk factors associated with the highest predictive power of mortality in hemodialysis, as evidenced by the meta-analysis of Herselman and colleagues $[10,14]$. The fact that underweight is linked to increased mortality is consistent with the "obesity paradox" posed by Vashistha et al. in a vast cohort of 123.383 patients undergoing hemodialysis. This study established an inverse relationship between BMI and the probability of death [15].

Is worth mentioning that although the cases had a higher proportion of catheter use vs controls, and both groups were far from the targets prescribed by the KDIGO 2012 Guidelines and the DOOPS record (catheters in $<10 \%$ of patients), this factor had no impact on the mortality outcome $[3,16]$. In the present study, catheter use and anemia did not reach a statistically significant difference in relation to variables adjustments despite clinical significance of a greater proportion of patients in cases than in controls. These factors are important to keep in hand, considering the association with cardiovascular mortality evidenced in other studies [17-20]. 
It is noteworthy that several traditional and non-traditional risk factors associated with mortality, and widely evidenced in other studies i.e., hypertension, diabetes mellitus and a history of established cardiovascular disease, anemia, intradialytic hypotension, dyslipidemia and hyperphosphatemia and the rate of cardiovascular morbidity and mortality Kt/V $<1.4$ (24), did not have any statistical significance in the present study [17,18, 21,22].

Regarding the causes of death of the cases, cardiovascular was the prevailing cause. This was consistent with the DOOPS and the National Statistics records $[2,3]$.

This study experienced several limitations. First, it was impossible to establish causality because of the retrospective design. However, it is worth noting that the strict inclusion criteria used and the high OR values with a narrow CI indicate a high probability of association between hypoalbuminemia and low weight with mortality in hemodialysis. A study to quantify other factors related to mortality, such as C-reactive protein, hemoglobin A1C diabetes, left ventricular hypertrophy, hyperhomocysteinemia, interleukins 1 and 6 and cardiac troponin biomarkers, BNP and pro-T- BNP, which were not included in the present study could help define the validity of this result. With these factors further studies could be designed to measure their impact on mortality in our patient population in both hemodialysis and peritoneal dialysis $[23,24]$.

\section{Conclusions}

Patients on Hemodialysis treatment in Cartagena, Colombia, have a high prevalence of traditional and non-traditional risk factors. However, only underweight and Hypoalbuminemia were associated with increased mortality. It was found as well that the main cause of death is cardiovascular. Further studies are necessary to improve understanding of the association of variables with clinical outcomes and to determine how their intervention could influence reducing morbidity and mortality of these patients.

\section{References}

1. Crawford PW, Lerma EV (2008) Treatment options for end stage renal disease. Prim Care 35: 407-432.

2. Acuña L, Soler L, Sanchez P, Martínez D (2013) High cost account: Situation of chronic kidney disease in Colombia 2012. Ministry of Social Protection, Bogotá.

3. Goodkin D, Bragg-Gresham J, Koenig K (2003) Association of comorbid conditions and mortality in hemodialysis patients in Europe, Japan, and the United States: The dialysis outcomes patterns study (DOPPS). J Am Soc Nephrol 14: 3270-3277.

4. Badve SV, Roberts MA, Hawley CM, Cass A, Garg AX, et al. (2011) Effects of beta-adrenergic antagonists in patients with chronic kidney disease: a systematic review and meta-analysis. J Am Coll Cardiol 58: 1152-1161.

5. Matsumoto Y, Mori Y, Kageyama S, Arihara K, Sugiyama T, et al. (2014) Spironolactone reduces cardio- and cerebrovascular morbidity and mortality in hemodialysis patients. J Am Coll Cardiol 63: 528-536.

6. Trimarchi H, Muryan A, Dicugno M, Young P, Forrester M, et al. (2012) Proteinuria: An ignored marker of inflammation and cardiovascular disease in chronic hemodialysis. Int J Nephrol Renovasc Dis 5: 1-7.
7. Sanabria M, Muñoz J, Trillos C, Hernández G, Latorre C, et al. (2008) Dialysis outcomes in Colombia (DOC) study: a comparison of patient survival on peritoneal dialysis vs hemodialysis in Colombia. Kidney Int Suppl: S165-172.

8. Coronado Daza J, Luján Agamez M (2009) Prevalence of cardiovascular risk factors in patients with dialytic chronic kidney disease in the renal units of the city of Cartagena in the period 2004-2007. Rev ASOCOLNEF 1: 18-23.

9. US Renal Data System (2013) Annual Data Report: Atlas of Chronic Kidney Disease and End-Stage Renal Disease in the United States. National Institutes of Health, National Institute of Diabetes and Digestive and Kidney Diseases. Bethesda, USA.

10. Herselman M, Esau N, Kruger JM, Labadarios D, Moosa MR (2010) Relationship between serum protein and mortality in adults on long-term hemodialysis: Exhaustive review and meta-analysis. Nutrition 26: 10-32.

11. Lázaro L, Pereira C, Mora González S, Artimes Hernández Y (2005) Impacto de la malnutrición sobre la mortalidad en los pacientes en hemodiálisis crónica. Rev Cubana Med Milit 34: 1-9.

12. Dalas M, Vásquez A, Fernández Y, Guerra G (2008) Comportamiento de los factores pronósticos de morbilidad y mortalidad en una Unidad de Hemodiálisis. Rev Cuba Med 47: 1-12.

13. Broumand B (2007) Diabetes: changing the fate of diabetics in the dialysis unit. Blood Purif 25: 39-47.

14. Kubrusly M, Oliveira CM, Santos DC, Mota RS, Pereira ML (2012) A comparative analysis of pre- and post-dialysis albumin as indicators of nutritional and morbi-mortality risks in hemodialysis patients. J Bras Nefrol 34: 27-35.

15. Vashistha T, Mehrotra R, Park J, Streja E, Dukkipati R, et al. (2014) Effect of age and dialysis vintage on obesity paradox in long-term hemodialysis patients. Am J Kidney Dis 63: 612-622.

16. Eknoyan G, Lameire N (2013) KDIGO 2012 clinical practice guideline for the evaluation and management of chronic kidney disease. Kidney Int Suppl 3: 1-150.

17. Shastri S, Sarnak MJ (2010) Cardiovascular disease and CKD: core curriculum 2010. Am J Kidney Dis 56: 399-417.

18. Vlagopoulos PT, Tighiouart H, Weiner DE, Griffith J, Pettitt D, et al. (2005) Anemia as a risk factor for cardiovascular disease and all-cause mortality in diabetes: the impact of chronic kidney disease. J Am Soc Nephrol 16: 3403-3410.

19. Polkinghorne KR, McDonald SP, Atkins RC, Kerr PG (2004) Vascular access and all-cause mortality: a propensity score analysis. J Am Soc Nephrol 15: 477-486.

20. Tordoir J, Canaud B, Haage P, Konner K, Basci A, et al. (2007) EBPG on vascular access. Nephrol Dial Transplant 22: ii88-ii117.

21. Restrepo Valencia CA, Cruz J (2008) Safety and effectiveness of nicotinic acid in the management of patients with chronic renal disease and hyperlipidemia associated to hyperphosphatemia. Nefrologia 28: 61-66.

22. Nieto I, Mahecha P (2005) Asociación entre hiperfosfatemia y mortalidad en los pacientes con insuficiencia renal crónica terminal en terapia de reemplazo de la función renal con diálisis peritoneal ó hemodiálisis. Un estudio de la Unidad Renal de Villavicencio 1999-2003. Act Med Colomb 30: 1-5.

23. Apple F, Murakami M, Pearce L (2004) Multi-biomarker risk stratification of n-terminal pro-b-type natriuretic peptide, high-sensitivity c-reactive protein, and cardiac troponin $\mathrm{t}$ and $\mathrm{i}$ in end-stage renal disease for allcause death. Clinical Chemistry 50: 2279-2285.

24. Wang AY, Lai KN (2008) Use of cardiac biomarkers in end-stage renal disease. J Am Soc Nephrol 19: 1643-1652. 